\title{
Optimization of Emulsion Polymerization for Submicron-Sized Polymer Colloids towards Tunable Synthetic Opals
}

\author{
Seulgi Kim, Young Gon Seo, Youngjin Cho, Jinsub Shin, Seung Chul Gil, and Wonmok Lee* \\ Department of Chemistry, Sejong University, Seoul 143-747, Korea. *E-mail: wonmoklee@sejong.ac.kr \\ Received September 14, 2009, Accepted May 4, 2010
}

\begin{abstract}
Submicron-sized polymeric colloidal particles can self assemble into 3-dimensional (3D) opal structure which is a useful template for photonic crystal. Narrowly dispersed polymer microspheres can be synthesized by emulsion polymerization in water using water-soluble radical initiator. In this report, we demonstrate a facile and reproducible emulsion polymerization method to prepare various polymeric microspheres within $200-400 \mathrm{~nm}$ size ranges which can be utilized as colloidal photonic crystal template. By controlling the amount of monomer and surfactant, monodisperse polymer colloids of polystyrene (PS) and acrylates with various sizes were successfully prepared without complicated synthetic procedures. Such polymer colloids self-assembled into 3D opal structure exhibiting bright colors by reflection of visible light. The colloidal particles and the resulting opal structures were rigorously characterized, and the wavelength of the structural color from the colloidal crystal was confirmed to have quantitative relationship with the size of constituting colloidal particles as predicted by Bragg equation. The tunability of the structural color was achieved not only by varying the particle size but also by infiltration of the colloidal crystal with liquids having different refractive indices.
\end{abstract}

Key Words: Photonic crystals, Self-assembly, Emulsion polymerization, Colloids, Opal

\section{Introduction}

Photonic crystal is a dielectric material having a submicron size order periodicity in the lattice structure through which the light of a specific wavelength range cannot propagate. Such a photonic property is often called "photonic bandgap" since it is analogous to the electronic bandgap for semi-conductors. ${ }^{1-2}$ Utilizing the photonic crystal as a platform material, the flow of light can be controlled, and thus various photonic devices can be realized such as micro-laser, ${ }^{3-4}$ no-loss waveguide ${ }^{5-7}$ and chemical sensors. ${ }^{8-9}$ The fabrication methods for photonic crystal are categorized to 1) top-down approach such as photolithography ${ }^{10-11}$ and diffraction lithography, ${ }^{12-13}$ and 2) bottom-up approach such as colloidal self-assembly, ${ }^{14-21}$ and multi-photon polymerization method. $^{22-23}$ Among those techniques, selfassembled colloidal crystal has attracted much attention since it shows multiple advantages such as a relative simplicity and cost-effectiveness towards the fabrication process of large area $3 \mathrm{D}$ photonic crystal template. In order to ensure practical applicability of colloidal crystals, however, high quality colloidal microspheres with a controlled size and a size distribution are essentially required. A dispersion of colloidal microsphere can self-assemble into the 3D closed packed structure (i.e., face centered cubic) with various domain sizes and random defect densities depending on the size distribution of colloidal system and the crystallization conditions. Indication of a successful colloidal crystallization is a characteristic color, so called 'structural color' which comes from the constructive interference of visible light of specific wavelength range due to existence of periodic structure in the colloidal crystal. Such a structural color is often called 'stop band' from photonics point of view. The monodisperse polymeric microspheres of photonic crystal grade can be obtained by emulsion polymerization technique. ${ }^{24}$ Emulsion polymerization is relatively simple and versatile since various monomers are applicable which can be radically polymerized through addition mechanism. There have been a lot of studies on emulsion polymerization from various points of view such as size control, functionality control, cost, and so on. ${ }^{24-26} \mathrm{Re}-$ cently, Egen et al. have reported a simple emulsion polymerization method without using an amphiphilic surfactant otherwise necessary for controlling the size of microspheres. ${ }^{24}$ However, comprehensive investigations including the synthesis, the colloidal crystallization, and the optical characterizations are hardly found.

In this report, we demonstrate a simple and reproducible emulsion polymerization of microspheres of various monomer systems having $100 \sim 400 \mathrm{~nm}$ size range and narrow distribution. Taking the technical importance into account, we focus on the emulsion polymerization of poly(methyl methacrylate) (PMMA), poly(t-butyl methacrylate) (PtBMA), and polystyrene (PS) ${ }^{25}$ All three polymers are transparent to visible light, and have their respective importance in various photonic applications. Rigorous characterizations on the microspheres are performed from the mechanistic point of view. A simple fabrication method of colloidal photonic crystals from those microspheres, and their optical characterizations are also investigated.

\section{Experimental}

Materials. Methyl methacrylate (MMA), $t$-butyl methacrylate ( $t$ BMA), potassium persulfate (PPS), sodium dodecylsulfate (SDS) were purchased from Aldrich, and used as received. Styrene was purified with activated alumina (basic, Aldrich). Deionized(DI) water was obtained from a water purifier (Power I Plus, Human Technology) equipped with ion exchange resin, and used for purification and preparation of aqueous polymer dispersions. After polymer microsphere is purified, ion exchange resin (AG501-X8, Bio-Rad) was used for preservation of 
colloidal dispersions.

Polymerization of methacrylates. Poly(methyl methacrylate) (PMMA) and poly(t-butyl methacrylate) (PtBMA) colloidal particles were synthesized by surfactant-free emulsion polymerization (SFEP) by modifying the method reported by Egen et al. $^{24}$ Thermally dissociable initiator, PPS was dissolved in DI water and transferred to a $50 \mathrm{~mL}$ two-neck round bottomed flask, the temperature of which has been maintained at $90{ }^{\circ} \mathrm{C}$ in silicone oil bath. The aqueous initiator solution was purged with nitrogen $\left(\mathrm{N}_{2}, 99.99 \%\right.$, Donga Gas Inc.) for 30 min with stirring. MMA was quickly inserted using gas-tight syringe $(50 \mathrm{~mL}$, Hamilton) with long stainless steel needle. Emulsion polymerization was carried out for 1.5 hours under nitrogen atmosphere with vigorous stirring (at $1000 \mathrm{rpm}$ ). Upon completion of polymerization, the turbid PMMA dispersion was filtered through cotton-filled syringe to be free from polymer chunks, and purified by ultra-centrifugation several times at $12000 \mathrm{rpm}$ with change of DI water to remove unreacted initiator and monomer. Finally, $\sim 10$ weight $\%$ (wt \%) of aqueous colloidal dispersion was preserved in a refrigerator after addition of small amount of ion exchange resin. PtBMA was synthesized by the same procedure as that of PMMA only differing in the monomer.

Polymerization of PS. PS microsphere was synthesized by emulsion polymerization in the presence of surfactant. After purging temperature stabilized DI water under $\mathrm{N}_{2}$ flow for 1 hour, PPS as initiator and SDS as a surfactant were added followed by quick addition of styrene which had been filtered through activated alumina. After finishing polymerization at 70 ${ }^{\circ} \mathrm{C}$ for 4 hours, the same work-up step as described above was performed if particle size exceeds $250 \mathrm{~nm}$. However, PS particles smaller than $250 \mathrm{~nm}$ were purified by using semi-permeable membrane since they sediment too slowly due to a lower density than those of PMMA and P $t$ BMA. As synthesized polymer dispersion was filtered through pre-cleaned cotton fibers, it was poured to a semi-permeable cellulose membrane tube (MWCO 12000 - 14000, MFPI) with one side clipped. After clipping the other side, the emulsion-filled tube was fully soaked in $5 \mathrm{~L}$ beaker containing DI water. The low molecular weight impurities in the tube were removed in this manner. To speed up the purification process, DI water was freshly exchanged every 4 hours until the resistivity of DI water in the beaker reaches 10 Mohms. The final aqueous dispersions contained $\sim 10 \mathrm{wt} \%$ of the polymer microspheres.

Colloidal crystallization. About $0.1 \mathrm{~mL}$ of each colloidal dispersion containing $10 \mathrm{wt} \%$ of the microsphere were drop cast onto the microscope slide glass which was pre-cleaned with piranha solution (3:1 mixture of concentrated sulfuric acid and aqueous hydrogen peroxide) and DI water. The slide glass was placed in a glass petri dish with a cover where a cotton cloth fully soaked with water is placed beside the sample to slow down water evaporation from the colloidal dispersion. The colloidal crystallization slowly proceeds for 1-2 days. Upon complete drying in air, each colloidal crystal sample exhibited a bright structural color by selective reflection of room light, which implies the periodic structure of photonic crystal has been successfully developed.

Particle size analyses of colloidal microspheres. The average particle size of the colloidal dispersions were characterized

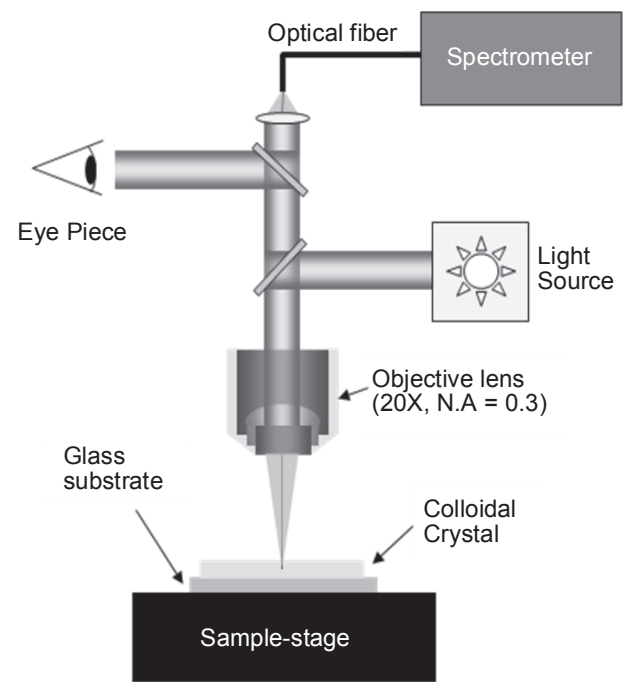

Figure 1. Schematic illustration of reflectance measurement.

using an electrophoretic light scattering analyzer (ELS 8000, Otsuka electronics Co.) which measures the particle size and the distribution by means of dynamic light scattering of the particle in water. Each emulsion was diluted with DI water to $\sim 0.1 \mathrm{wt} \%$, and directly subjected to the particle size analyzer.

Molecular weight analyses of PS colloids. The average molecular weights (M.W.) of colloidal particles were characterized by size exclusion chromatography (SEC) with dimethyl formamide (DMF) as an eluent. Each sample dissolved in DMF at $\sim 2 \mathrm{mg} / \mathrm{mL}$ concentration was injected to SEC apparatus (Younlin Inc.) equipped with two SEC columns (Mixed-B, Mixed-C, Polymer Lab), and the chromatogram was monitored by UV absorbance detector operated at $270 \mathrm{~nm}$. The M.W. and M.W. distribution was calculated by standard calibration with 7 PS standards (Polymer lab).

Characterization of colloidal crystals. The 3D structures of the fabricated opalline colloidal crystals were characterized by scanning electron microscopy (SEM) (S-4700, HITACHI). Each sample was dried in vacuum oven (LK Lab Korea) and sputter-coated with thin $(\sim 5 \mathrm{~nm}) \mathrm{Pt} / \mathrm{Pd}$ layer prior to the measurement. For the reflectance measurement of each colloidal crystal, a fiber optic UV-vis spectrometer (AvaSpec, Avantes) was used which was coupled with an reflected light microscope (Bimeince). The schematic illustration of the measurement system is shown in Figure 1. The colloidal crystal on slide glass was placed on the sample stage, where the white light from Tungsten lamp was focused via objective lens $(20 \mathrm{x}, \mathrm{NA}=0.3)$. Once the focused spot is found using eye piece, the reflected light from the sample was introduced though a taylor-made adaptor to the focusing lens of the optic fiber which guides the light into the spectrometer. The measured reflectance spectrum was obtained through a normalization by the reflectance spectrum of incident light captured from silver mirror. Although the ideal reflectance measurement assumes a purely normal incidence of light and the correspondingly normal reflection, our system measures the multiple reflectance spectra over the incidence angles of 0 to $17.5^{\circ}$ due to the numerical aperture (NA) of the objective lens. 


\section{Results and Discussion}

In the present study, we aimed to obtain the polymeric microspheres which can self-assembled into the colloidal crystals to reflect selective wavelength range of visible or near-IR light. Number of pioneering literatures have reported that the polymeric microspheres having the diameters in the range of 100 $400 \mathrm{~nm}$ fall in such "photonic grade" microspheres. ${ }^{14,16,24-25,27}$ We have confirmed that the polymer microspheres from the acrylic monomer such as MMA, $t$ BMA within the size of photonic grade could be conveniently synthesized without the use of surfactant which usually takes part in the emulsion polymerization. ${ }^{24}$ In SFEP, the initiation takes place by scission of potassium persulfate, followed by radical attacks to some monomers to form charged oligomers. Such oligomers behave as surfactants to stabilize monomer droplet (micelle), resulting in the growth of polymer microsphere in a similar manner as "classical" emulsion polymerization. ${ }^{28}$ The particle size of the microspheres could be precisely controlled by varying the monomer concentration in the reaction mixture. The particle size of PMMA and PtBMA is known to have linear relationship with logarithm of the monomer concentration $(\mathrm{M}){ }^{28}$ Such tendency is shown in Figure 2, and the detailed data for each microsphere are summarized in Table 1.

The experimental results in Table 1 show that the change of the particle size is attributed to the varying monomer concentration in water regardless of initiator concentration. According to

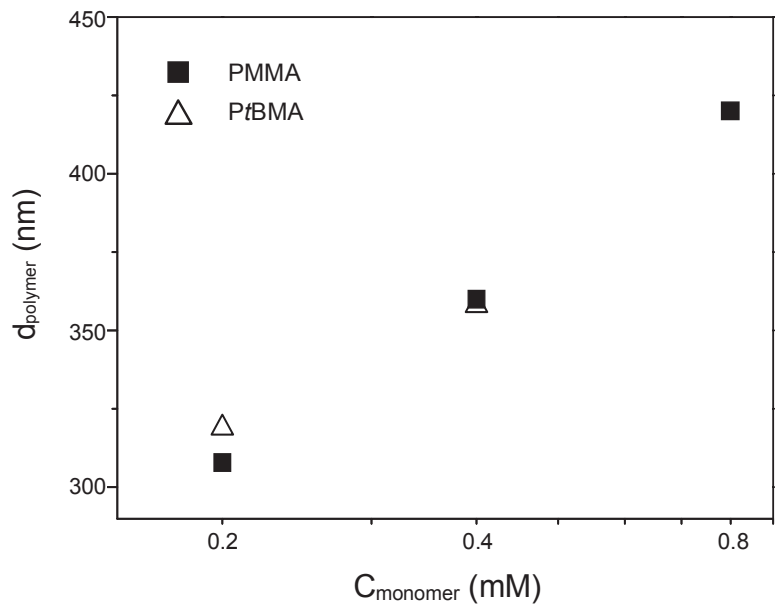

Figure 2. Relationship between monomer concentration and size (diameter) of PMMA and PtBMA microspheres.

Table 1. PtBMA and PMMA microspheres with various particle sizes

\begin{tabular}{ccccc}
\hline Sample & $\begin{array}{c}\text { Batch size } \\
{[\mathrm{mL}]}\end{array}$ & $\mathrm{C}_{\text {monomer }}[\mathrm{M}]$ & $\begin{array}{c}\mathrm{C}_{\text {initiator }} \\
{[\mathrm{mM}]}\end{array}$ & $\begin{array}{c}\mathrm{d}_{\text {polymer }} \\
{[\mathrm{nm}]}\end{array}$ \\
\hline PtBMA-319 & 18 & 0.2 & 12 & 319 \\
P $t$ BMA-358 & 18 & 0.4 & 12 & 358 \\
PMMA-308 & 20 & 0.2 & 3.5 & 308 \\
PMMA-360 & 20 & 0.4 & 7 & 360 \\
PMMA-420 & 20 & 0.8 & 7 & 420 \\
\hline
\end{tabular}

*Particle size was measured by dynamic light scattering a model ${ }^{27}$ the number of seeds (polymer micelle) at the initial stage of polymerization does not depend on the number of growing chains (initiator) but on the solubility of monomer. Therefore, regardless of initiator concentration, approximately the same number of polymer micelles starts to grow consuming monomers. A larger monomer concentration will result in a larger microspheres maintaining the number initially formed seeds. It is worthwhile to note that the relationship between the diameter of polymer microsphere $\left(\mathrm{d}_{\text {polymer }}\right)$ and the concentration of monomer $\left(\mathrm{C}_{\text {monomer }}\right)$ can be fitted by the following master curve both for PMMA and PtBMA.

$$
\mathrm{d}_{\text {polymer }}=186 \mathrm{~nm} \cdot \log \mathrm{C}_{\text {monomer }}+437 \mathrm{~nm}
$$

The fitting result is in a good agreement with those reported by Zentel and coworkers. ${ }^{24}$ Once the unreacted monomer and initiator are removed by centrifugation, the colloidal dispersions were successfully cast into the crystalline opal structure by slow evaporation of water on the slide glass.

As shown in Figure 3, the aqueous dispersion of the microsphere is cast on a glass substrate, and a controlled drying of the dispersion for 1 - 2 days typically resulted in shiny colloidal crystal films showing the characteristic structural color upon complete evaporation of water. It is well known that the drying meniscus induces the capillary force between microspheres which will be closely packed each other (Figure 3). Although there have been clever ideas to fabricate colloidal crystals over large area, the size distribution of the colloidal particle is the most important factor affecting the final quality of the colloidal

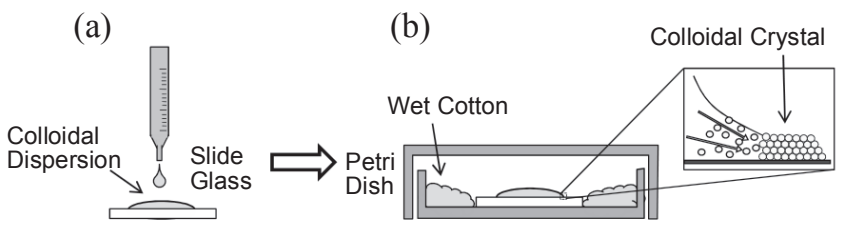

Figure 3. Colloidal crystallization of polymer dispersion by controlled water evaporation.

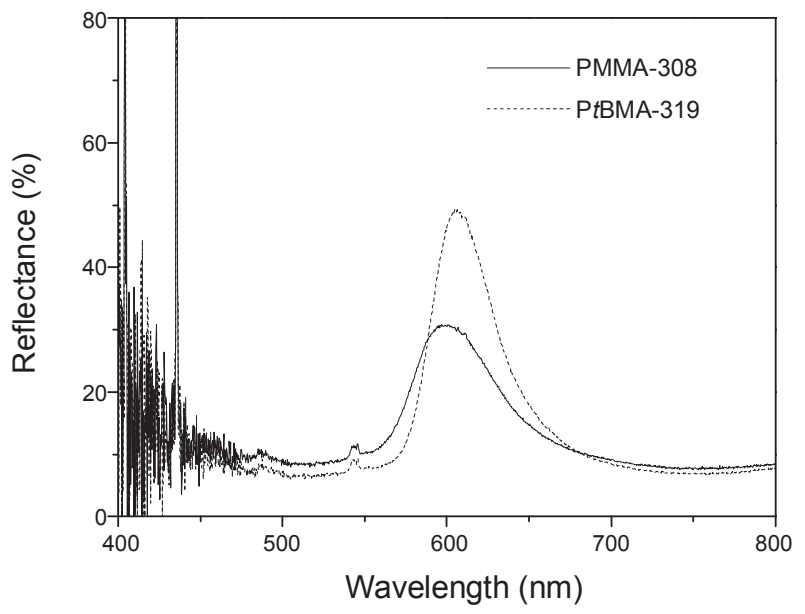

Figure 4. Reflectance spectra of visible light from a colloidal crystal fabricated from PMMA-308 and PtBMA-319. 
Table 2. PS microspheres synthesized in this study

\begin{tabular}{ccccccc}
\hline Sample & Batch size $[\mathrm{mL}]$ & $\mathrm{C}_{\text {monomer }}[\mathrm{M}]$ & $\mathrm{C}_{\text {initiator }}[\mathrm{mM}]$ & $\mathrm{C}_{\text {SDS }}[\mathrm{mM}]$ & $* \mathrm{~d}_{\text {polymer }}[\mathrm{nm}]$ & $* \mathrm{M}_{\mathrm{n}}[\mathrm{kg} / \mathrm{mol}]$ \\
\hline PS-420 & 18 & 3.0 & 10 & 0.06 & 420 & 25.7 \\
PS-350 & 18 & 3.0 & 10 & 0.1 & 350 & 36.0 \\
PS-290 & 18 & 3.0 & 10 & 0.2 & 290 & 42.0 \\
PS-240 & 18 & 3.0 & 10 & 0.4 & 240 & 36.4 \\
PS-210 & 18 & 3.0 & 10 & 0.6 & 210 & 65.2 \\
PS-180 & 18 & 3.0 & 10 & 2.0 & 180 & 163 \\
PS-115 & 18 & 3.0 & 10 & 0.8 & 129
\end{tabular}

*Particle size was measured by dynamic light scattering. ${ }^{*} \mathrm{M}_{\mathrm{n}}$ : Number averaged molecular weight measured by SEC.

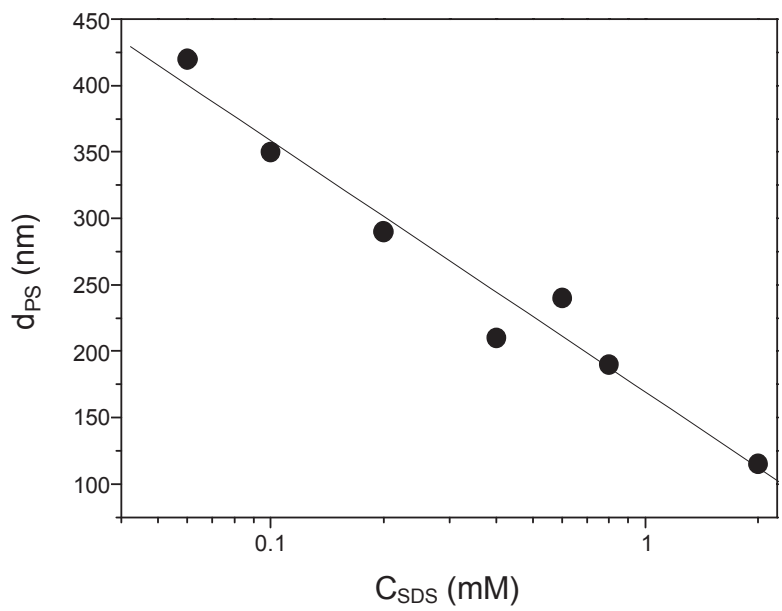

Figure 5. Relationship between the amount of surfactant and PS diameter.

crystal film. The size distributions of polymethacrylic microspheres were confirmed to be as narrow as $2.5 \%$ from the dynamic light scattering measurement. Narrow size distribution of the microsphere is important toward photonic crystal application since the particles of different sizes would generate random defects consequently deteriorating the quality of the colloidal photonic crystal. ${ }^{15,29-30}$ By simply casting the colloidal dispersions without sophisticated crystallization technique, the high quality colloidal crystals could be obtained showing the narrow size distributions of acrylic microspheres synthesized in this study.

Reflectance measurements on the colloidal crystals fabricated from the as-synthesized PMMA and P $t$ BMA clearly exhibited narrow stop bands in visible wavelength range as shown in Figure 4.

Although methacrylic monomers were successfully converted to "photonic grade" colloidal microspheres without using surfactant, emulsion polymerization of the styrene monomer following the same procedure usually ended up with the microspheres having $500-600 \mathrm{~nm}$ in diameter which are too large for photonic crystal application. In order to decrease the average diameter, a water soluble surfactant, SDS was introduced prior to the polymerization and the resultant PS microspheres were characterized. The details are compiled in Table 2.

Figure 5 shows the relationship between the PS particle's

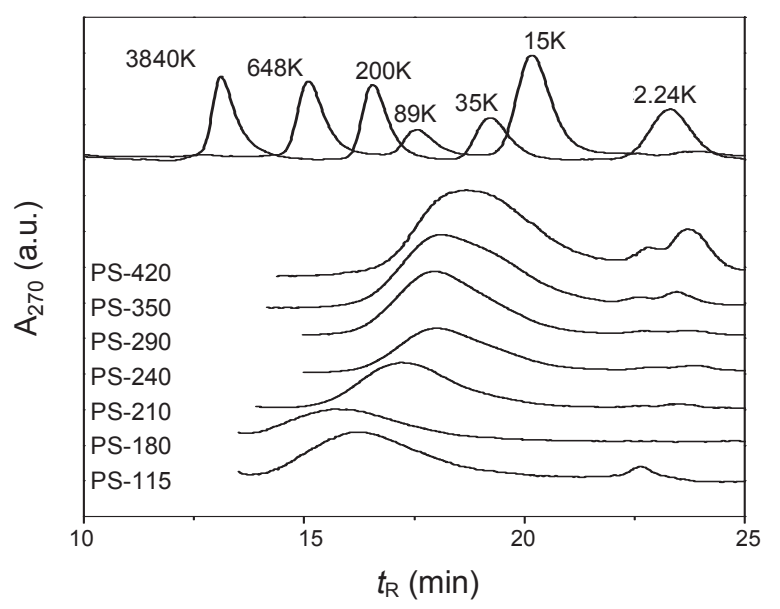

Figure 6. SEC Chromatograms of PS microsphere samples dissolved in DMF. The chromatograms of 7 PS standards are also shown (top chromatogram).
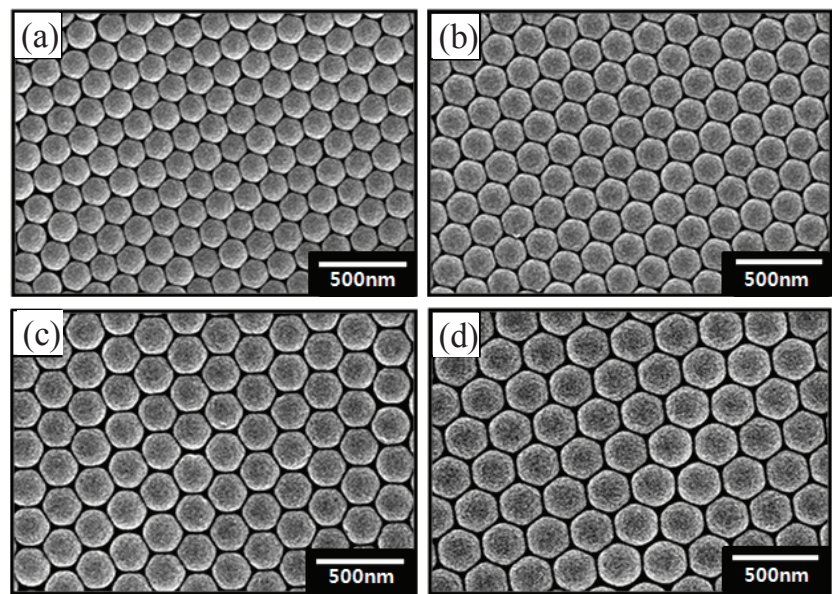

Figure 7. SEM images of the colloidal crystals fabricated from the PS particles of differential particle sizes. (a) PS-190, (b) PS-210, (c) PS-240, (d) PS-290.

diameter and the amount of surfactant for the emulsion polymerization. As expected, increased amount of SDS resulted in a decreased particle size due to an efficient stabilization of small sized monomer droplets by SDS during the polymerization. The plot in Figure 5 satisfies the following relationship as predicted in a conventional emulsion polymerization. ${ }^{28}$ 


$$
\mathrm{d}_{\mathrm{PS}}=-189 \mathrm{~nm} \cdot \log \mathrm{C}_{\mathrm{SDS}}+169 \mathrm{~nm}
$$

The decrease in the PS particle size with increased SDS concentration doesn't reflect the tendency of the molecular weight (M.W.) of PS in the particles. Actually, the emulsion polymerization theory predicts there will be an increase in number averaged molecular weight $\left(\mathrm{M}_{\mathrm{n}}\right)$ by adding more surfactant at the given initiator concentration. ${ }^{28}$ This is because the addition of surfactant causes a larger numbers of monomer micelles (particles) which will result in the generation of less numbers of radicals per particle during the polymerization to give a larger molecular weight.

As shown in Figure 6, we confirmed that $M_{n}$ of PS microspheres increases by increased amount of SDS through SEC analyses on the microsphere samples dissolved in DMF, and such opposite tendencies of the particle size and the molecular weight with an increase of SDS concentration are also summarized in Table 2.

Figure 7 shows the SEM images of the colloidal crystals viewed from their top surface. The different particle sizes according to the varying reaction condition and hexagonally packed [111] plane of FCC structure are evident from the SEM images. Although not shown, smaller particles below $100 \mathrm{~nm}$ exhibited broad size distribution due to a short growth period at the given nucleus during the emulsion polymerization. The practical size ranges of reproducible and monodisperse PS microspheres which can be synthesized by the emulsion polymerization method used in this study were thus found to be 200 to $400 \mathrm{~nm}$. A larger particle size should be achievable by changing reaction temperature or by introducing additional amount of monomer. PS colloidal crystals having $200 \sim 400 \mathrm{~nm}$ size range are known to show stop band at the visible wavelength by the specular reflection of incident light.

Figure 8 shows the colors and respective reflectance spectra of the PS colloidal crystals having different colloidal sizes. Due to the monodisperse size distribution of PS microspheres, the resulting colloidal crystals exhibited bright structural colors. The fringed patterns around peak maxima suggest that the exposed region for the reflection measurement is single crystalline phase. A closer look at the colors and the reflection spectra in Figure 8 reveals that the colloidal crystals from PS-350 and PS420 have the first order reflection maxima placed at $790 \mathrm{~nm}$ and $970 \mathrm{~nm}$ respectively which are the wavelengths beyond visible range. For those cases, apparent colors arise from higher order peaks which are weaker than those of the first order reflection. For the colloidal crystal from PS-420 in particular, higher order diffractions were assigned by arrows in the Figure 8 . The greenish color of PS-420 colloidal crystal as shown in Figure 8 comes from the mixed contribution of such higher order reflections centered at $480 \mathrm{~nm}$ and $560 \mathrm{~nm}$. The relationship between the size of microspheres in the opal structure and the wavelength of maximum reflection ( $\lambda_{\max }$, peak maxima of the stop band) can be easily derived from the first order Bragg reflection by [111] planes of FCC structure of the colloidal crystal and its filling factor $(0.74)$ as following. ${ }^{9}$

$$
\begin{aligned}
& \lambda_{\max }=(8 / 3)^{1 / 2} \cdot \mathrm{n}_{\text {eff }} \cdot d \\
& n_{\text {eff }}=\left(0.74 \cdot n_{\text {polymer }}+0.26 \cdot n_{\text {interstice }}{ }^{2}\right)^{1 / 2}
\end{aligned}
$$
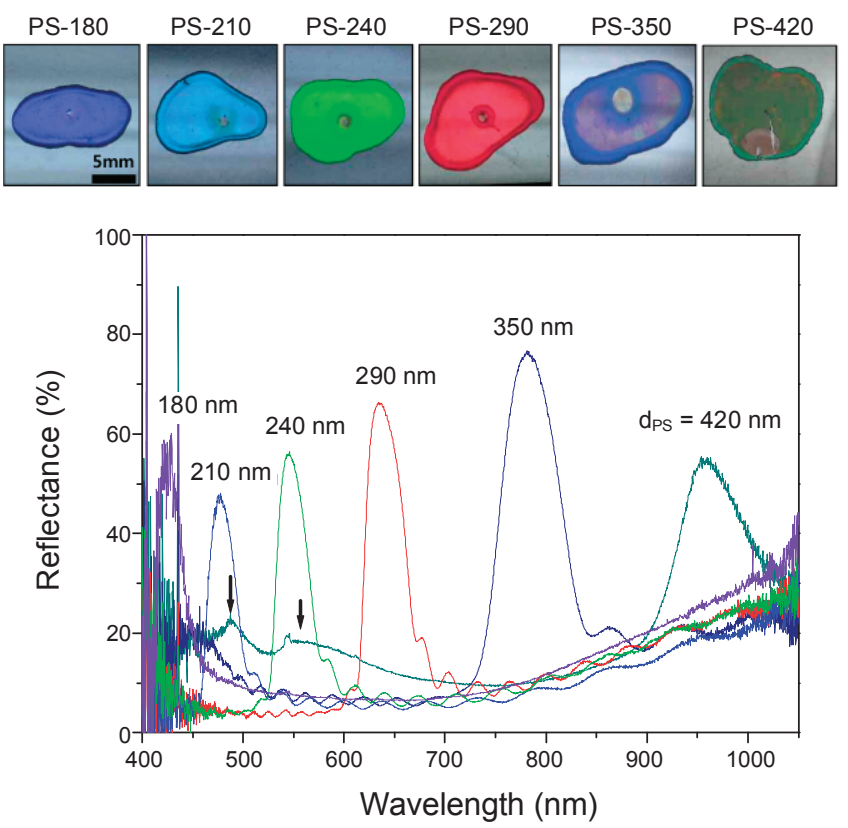

Figure 8. Colors and reflectance spectra of the colloidal crystals made from the PS's of different particle sizes. Arrows indicate higher order diffraction of PS-420 responsible for weak structural color.

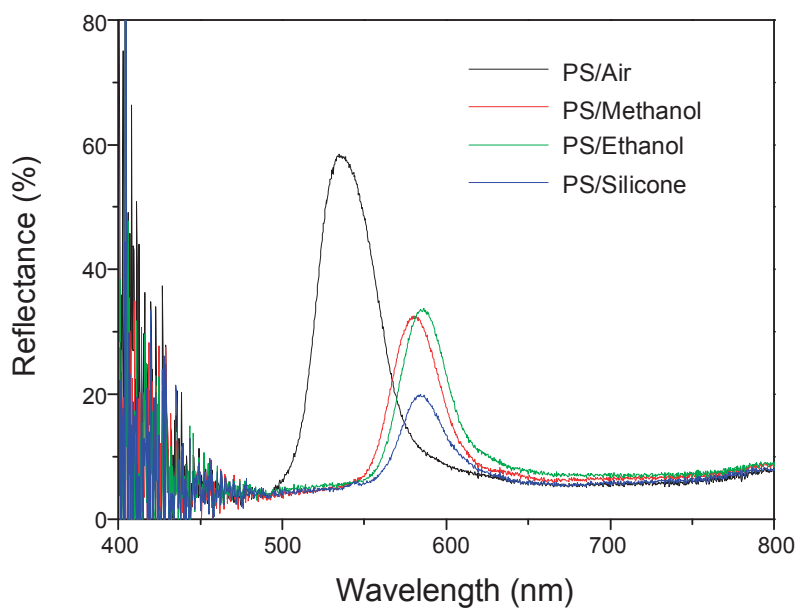

Figure 9. Reflectance spectra of PS $(d=230 \mathrm{~nm})$ colloidal crystals infiltrated with different media.

where $\mathrm{d}$ is a diameter of the constituting microsphere, $\mathrm{n}_{\mathrm{eff}}$ is an effective refractive index, and $\mathrm{n}_{\text {polymer }}$ and $\mathrm{n}_{\text {interstice }}$ denote refractive index of polymeric microsphere and interstice material respectively. Therefore, approximation of the microsphere size is possible by measuring $\lambda_{\max }$ of the corresponding colloidal crystal, and applying Bragg relation. Although not shown, the calculated and the measured diameters for the PS microspheres showed a good accordance.

Wavelength tuning of the reflected colors of colloidal crystal can be achieved not only by size control of colloidal particles but also by infiltration of interstitial spaces of colloidal crystals with transparent materials. In Figure 9, reflectance spectra of PS colloidal crystal $\left(\mathrm{d}_{\mathrm{PS}}=230 \mathrm{~nm}\right)$ filled with different liquids are shown. Initially, the interstitial volume was occupied by air 
Table 3. Comparison of reflection maxima of PS colloidal crystals filled with different materials

\begin{tabular}{lcccc}
\hline & PS/Air & PS/Methanol PS/Ethanol & PS/Silicone \\
\hline$\lambda_{\text {max, measured }(\mathrm{nm})}$ & 535 & 580 & 590 & 587 \\
$\lambda_{\text {max, calculated }}(\mathrm{nm})$ & 548 & 573 & 576 & 580 \\
Mismatch (\%) & -2.4 & 1.2 & 2.4 & 0.5 \\
\hline
\end{tabular}

$(\mathrm{n}=1.00)$, and it has been infiltrated by methanol $(\mathrm{n}=1.328)$, ethanol $(\mathrm{n}=1.361)$ and silicone elastomer (Sylgard 184, Dow corning, $n=1.404$ ) respectively. The infiltration of air void with a higher index material caused a red shift in $\lambda_{\max }$. This can be easily understood by the increased $n_{\text {eff }}$ in Bragg equation. One can calculate the predicted $\lambda_{\max }$ using the particle diameters and the refractive indices of the materials (i.e., $\left.\mathrm{n}_{\mathrm{PS}}=1.590\right)^{31}$ and compare them with the measured $\lambda_{\max }$. Table 3 shows the comparison between the measured and the calculated $\lambda_{\max }$ by Bragg equation as mentioned above. The discrepancy between the measured and the calculated $\lambda_{\max }$ was $-2.4 \%$ for PS/Air, while those for the infiltrated colloidal crystals were $0.5 \sim 2.4 \%$. In practice, there are several possible sources of error in the calculation such as the accuracy issues in the microsphere diameter, refractive index and filling factor. The positive values of mismatch for the infiltrated samples explain that the infiltration caused a small degree of red-shift in the measured $\lambda_{\max }$ in comparison to the calculation, which can be attributed to the swelling of PS particles during the infiltration of liquid of Silicone precursor. Taking all those factors into account, however, our calculation showed a pretty good agreement with the measured $\lambda_{\max }$. In addition to the red shifts in $\lambda_{\max }$, infiltration also caused the decrease in the reflectance as shown in Figure 9. By the photonic bandgap theory, reflectance is strongly dependent on the ratio of the refractive indices of two materials $\left(\mathrm{n}_{\mathrm{r}}=\mathrm{n}_{\text {high }} / \mathrm{n}_{\text {low }}\right)$ which is why high index material is required to achieve 'complete' $3 \mathrm{D}$ photonic bandgap showing $100 \%$ reflectance at the wavelength of interest. ${ }^{20}$ For instance, $\mathrm{n}_{\mathrm{r}}$ greater than 3.0 is necessary for FCC lattice. ${ }^{20}$ In our case, decreased $\mathrm{n}_{\mathrm{r}}$ by infiltration is responsible for the decrease in reflectance, and the lowest $n_{r}$ by PS/Silicone showed the weakest reflectance.

As demonstrated so far, the monodisperse polymeric microspheres made in this study can be successfully utilized as platform materials for photonic crystal applications. In conjunction with various fabrication techniques of colloidal crystal as well as infiltration of functional nanomaterials, developments of novel photonic crystal devices such as chemical sensors, dye sensitized solar cell are underway.

In summary, we have synthesized the monodisperse microspheres of PMMA, P $t$ BMA, and PS through simple emulsion polymerization. We confirmed that the particle sizes of PMMA and $\mathrm{P} t \mathrm{BMA}$ are linearly increased proportional to monomer concentration in the reaction mixture. The particle size of PS showed inversely proportional relationship with the surfactant concentration. All the polymeric microspheres showed monodisperse size distributions (standard deviation $<2.5 \%$ ) without complicated size selection procedures. A slow drying of water resulted in the formation of opal structure via self-assembly process, which exhibited respective colors by reflection of visible light according to the particle size. The particle size of the colloidal microspheres could be also derived by applying Bragg relationship to the reflectance maxima measured from the corresponding colloidal crystals.

Acknowledgments. This work was supported by the Korea Research Foundation Grant funded by the Korean Government (MOEHRD, Basic Research Promotion Fund) (KRF-2008-331D00153).

\section{References}

1. Joannopoulos, J. D.; Meade, R. D.; Winn, J. N. Photonic Crystals: Molding the Flow of Light; Princeton University Press: Princeton, 1995.

2. John, S. Phys. Rev. Lett. 1987, 58, 2486.

3. Noda, S.; Chutinan, A.; Imada, M. Nature 2000, 407, 608.

4. Shkunov, M. N.; DeLong, M. C.; Raikh, M. E.; Vardeny, Z. V.; Zakhidov, A. A.; Barughman, R. H. Synthetic Matals 2001, 116 , 485.

5. Braun, P. V.; Rinne, S. A.; Garcia-Santamaria, F. Adv. Mater. 2006, 18,2665

6. Lee, W.; Pruzinsky, S. A.; Braun, P. V. Adv. Mater. 2002, 14, 271.

7. Pruzinsky, S. A.; Braun, P. V. Adv. Func. Mater. 2005, 15, 1995.

8. Lee, K.; Asher, S. A. J. Am. Chem. Soc. 2000, 122, 9534.

9. Lee, Y. J.; Braun, P. V. Adv. Mater. 2003, 15, 563.

10. Lin, S.-Y.; Chow, E.; Hietala, V.; Villeneuve, P. R.; Joannopoulos, J. D. Science 1998, 282, 274.

11. Noda, S.; Tomoda, K.; Yamamoto, N.; Chutinan, A. Science 2000, 289,604

12. Campbell, M.; sharp, D. N.; Harrison, M. T.; Denning, R. G.; Turberfield, A. J. Nature 2000, 404, 53.

13. Jeon, S.; Park, J. U.; Cirelli, R.; Yang, S. M.; Heitzman, C. E.; Braun, P. V.; Kenis, P. J. A.; Rogers, J. A. Proc. Natl. Acad. Sci. USA 2004, 101, 12428.

14. Griesebock, B.; Egen, M.; Zentel, R. Chem. Mater. 2002, 14, 4023.

15. Lee, W.; Chan, A.; Bevan, M. A.; Lewis, J. A.; Braun, P. V. Langmuir 2004, 20, 5262.

16. van Blaaderen, A.; Ruel, R.; Wiltzius, P. Nature 1997, 385, 321.

17. Jiang, P.; Hwang, K. S.; Mittleman, D. M.; Bertone, J. F.; Colvin, V. L. J. Am. Chem. Soc. 1999, 121, 11630.

18. Jiang, P.; Ostojic, G. N.; Narat, R.; Mittleman, D. M.; Colvin, V. L. Adv. Mater. 2001, 13, 389.

19. Velev, O. D.; Kaler, E. W. Adv. Mater. 2000, 12, 531.

20. Vlasov, Y. A.; Bo, X. Z.; Sturm, J. C.; Norris, D. J. Nature 2001, $414,289$.

21. Xia, Y.; Gates, B.; Yin, Y.; Lu, Y. Adv. Mater. 2000, 12, 693.

22. Albota, M.; Beljonne, D.; Bredas, J.-L.; Ehrlich, J. E.; Fu, J.-Y.; Heikal, A. A.; Hess, S. E.; Kogej, T.; Levin, M. D.; Marder, S. R.; McCord-Maughon, D.; Perry, J. W.; Rockel, H.; Rumi, M.; Subramaniam, G.; Webb, W. W.; Wu, X.-L.; Xu, C. Science 1998, 281, 1653.

23. Sun, H.-B.; Kawakami, T.; Xu, Y.; Ye, J.-Y.; Matuso, S.; Misawa, H.; Miwa, M.; Kaneko, R. Opt. Lett. 2000, 25, 1110.

24. Egen, M.; Zentel, R. Chem. Mater. 2002, 14, 2176.

25. Caruso, F. Colloids and Colloid Assemblies; Synthesis, Modification, Organization and Utilization of Colloid Particles; WileyVCH: Weinheim, 2003.

26. Dong, H.; Lee, S. Y. Macromol. Res. 2009, 17, 397.

27. Goodwin, J. W.; Hearn, J.; Ottewill, R. H. Colloid Polym. Sci. 1974, 252, 464.

28. Odian, G. Principles of Polymerization, 3rd ed.; John Wiley and Sons: New York, 1991.

29. Checoury, X.; Enoch, S.; Lopez, C.; Blanco, A. Appl. Phys. Lett. 2007, 90, 161131

30. Lee, W.; Braun, P. V. Mater. Sci. Eng. C 2007, 27, 961.

31. Ma, X.; Lu, J. Q.; Brock, R. S.; Jacobs, K. M.; Yang, P.; Hu, X. Phys. Med. Biology 2003, 48, 4165. 